\title{
NOTE ON AN EIGENVALUE PROBLEM FOR AN ODE ORIGINATING FROM A HOMOGENEOUS P-HARMONIC FUNCTION
}

\author{
MURAT AKMAN, JOHN LEWIS, AND ANDREW VOGEL \\ Dedicated to V.P. Maz'ya on the occasion of his 80th birthday
}

\begin{abstract}
We discuss what is known about homogeneous solutions $u$ to the $p-$ Laplace equation, $p$ fixed, $1<p<\infty$, when $(A) u$ is an entire $p$ - harmonic function on Euclidean $n$ space, $\mathbb{R}^{n}$, or $(B) u>0$ is $p$ - harmonic in the cone,

$$
K(\alpha)=\left\{x=\left(x_{1}, \ldots, x_{n}\right): x_{1}>\cos \alpha|x|\right\} \subset \mathbb{R}^{n}, n \geq 2,
$$

with continuous boundary value zero on $\partial K(\alpha) \backslash\{0\}$ when $\alpha \in(0, \pi]$.

We also outline a proof of our new result concerning the exact value, $\lambda=1-$ $(n-1) / p$, for an eigenvalue problem in an ODE associated with $u$ when $u$ is pharmonic in $K(\pi)$ and $p>n-1$. Generalizations of this result are stated. Our result complements work of Krol' - Maz'ya for $1<p \leq n-1$.
\end{abstract}

\section{INTRODUCTION}

In this paper we first discuss what is known about homogeneous solutions $u$ to the p-Laplace equation, $p$ fixed, $1<p<\infty$, when $(A) u$ is an entire p-harmonic function on Euclidean $n$ space, $\mathbb{R}^{n}$, or $(B) u>0$ is p-harmonic in the cone,

$$
K(\alpha)=\left\{x=\left(x_{1}, \ldots, x_{n}\right): x_{1}>\cos \alpha|x|\right\} \subset \mathbb{R}^{n}, n \geq 2,
$$

with continuous boundary value 0 on $\partial K(\alpha)$. More specifically, $u$ is for fixed $p, 1<$ $p<\infty$, a solution to

$$
\nabla \cdot\left(|\nabla u|^{(p-2)} \nabla u\right)=0 \text { in } \mathbb{R}^{n} \text { or } K(\alpha)
$$

and

$$
u(t x)=t^{\lambda} u(x) \text {, for some real } \lambda \text { whenever } t>0 \text { and } x \text { is in the domain of } u \text {. }
$$

Given $x \in \mathbb{R}^{n} \backslash\{0\}$ introduce spherical coordinates $r=|x|, x_{1}=r \cos \theta, 0 \leq \theta \leq \pi$. If $u$ as in (1.2) is p-harmonic in $K(\alpha)$ and $u(1,0, \ldots, 0)=1$, then it turns out that $u$ has the additional form:

$$
u(x)=u(r, \theta)=r^{\lambda} \phi(\theta), r>0,0 \leq \theta<\alpha, \text { with } \phi(0)=1, \phi(\alpha)=0,
$$

for some $\lambda \in(-\infty, \infty)$ and $\phi \in C^{\infty}([0, \alpha])$.

2010 Mathematics Subject Classification. Primary 35P99; Secondary 76B15, 35Q35.

Key words and phrases. p-Laplacian, boundary Harnack inequality, homogeneous p-harmonic functions, eigenvalue problem. 
1.1. WHAT IS KNOWN. Case (A): Entire Solutions: In $\mathbb{R}^{2}$, Krol' in [12] constructed homogeneous p-harmonic functions, $u$, as in (1.3) with continuous boundary value 0 on $\partial K(\alpha)$ whenever $\alpha \in(0, \pi]$. If $\alpha=\pi /(2 k), k=$ a positive integer, one can use Schwarz reflection to extend $u$ to an entire $p$ - harmonic function in $\mathbb{R}^{2}$. Moreover in [17], the second author showed there are no real polynomial solutions to the pLaplace equation in $\mathbb{R}^{2}$ when $1<p<\infty$. Tkachev in [25] proved for $1<p<\infty, p \neq 2$, that there are no real homogeneous polynomials of degree three in $\mathbb{R}^{n}, n \geq 3$, that are solutions to the $p$ - Laplace equation. The second and third authors in [20] showed there are no real homogeneous polynomial solutions of degree four in $\mathbb{R}^{n}, n \geq 3$, and none of degree 5 in $\mathbb{R}^{3}$ to the $p$ - Laplace equation when $1<p<\infty, p \neq 2$. Finally recent work has been done by Tkachev in [26]. This paper contains some very interesting examples of $p$ harmonic and related functions but still no examples of $p-$ harmonic real polynomials when $p>1, p \neq 2$. In short we do not know of any entire homogeneous real solutions to the $p$ - Laplace equation of the form (1.2) in $\mathbb{R}^{n}$ when $1<p<\infty, p \neq 2$, other than those constructed by Krol' in two dimensions.

Case (B), Solutions in a Cone: It was first shown by Krol' and Maz'ya in [13] that if $1<p \leq n-1$ and $\alpha \in(0, \pi)$, is near enough $\pi$, then there exists a unique solution to (1.1) in $K(\alpha)$ of the special form (1.3) with $\lambda(\alpha)>0$. Tolksdorf in [27] showed that given $\alpha \in(0, \pi)$, there exist unique $\lambda_{i}, \phi_{i}, i=1,2$, with $\lambda_{2}<0<\lambda_{1}$, and $\phi_{i}$, infinitely differentiable on $[0, \alpha]$ satisfying $\phi_{i}(\alpha)=0, \phi_{i}(0)=1$, and $u_{i}(r, \theta)=r^{\lambda_{i}} \phi_{i}(\theta), i=1,2$, are solutions to the $p$ - Laplace equation in $K(\alpha)$. Also Porretta and Véron gave another proof of Tolksdorf's result in [24]. A similar study was made in more general Lipschitz cones by Gkikas and Véron in [8].

Now we discuss what is known about eigenvalues $\lambda$ in (1.3) for various $\alpha, n$. Krol' and Krol' and Maz'ya in the papers mentioned above (see also [4]), used (1.1) to show for $u$ as in (1.3) that

$$
\begin{aligned}
& 0=\frac{d}{d \theta}\left\{\left[\lambda^{2} \phi^{2}(\theta)+\left(\phi^{\prime}\right)^{2}(\theta)\right]^{(p-2) / 2} \phi^{\prime}(\theta)(\sin \theta)^{n-2}\right\}+ \\
& \lambda[\lambda(p-1)+(n-p)]\left[\lambda^{2} \phi^{2}(\theta)+\left(\phi^{\prime}\right)^{2}(\theta)\right]^{(p-2) / 2} \phi(\theta)(\sin \theta)^{n-2}
\end{aligned}
$$

Letting $\psi=\phi^{\prime} / \phi$ in the above equation Krol' in [12] obtained, the first order DE

$$
\begin{aligned}
& 0=\left((p-1) \psi^{2}+\lambda^{2}\right) \psi^{\prime} \\
& +\left(\lambda^{2}+\psi^{2}\right)\left[(p-1) \psi^{2}+(n-2) \cot \theta \psi+\lambda^{2}(p-1)+\lambda(n-p)\right]
\end{aligned}
$$

If $n=2$ the cotangent term in the above DE goes out and variables can be separated in (1.4) to get

$$
\frac{\lambda d \psi}{\lambda^{2}+\psi^{2}}-\frac{(\lambda-1) d \psi}{\lambda^{2}+\psi^{2}+\lambda(2-p) /(p-1)}+d \theta=0 .
$$

The boundary conditions imply that $\phi$ is decreasing on $(0, \alpha)$ so $\psi(\alpha)=-\infty, \psi(0)=$ 0 . Using this fact and integrating it follows that

$$
\pm 1-\frac{\lambda-1}{\sqrt{\lambda^{2}+\lambda(2-p) /(p-1)}}=2 \alpha / \pi
$$


where +1 is taken if $\lambda>0$ and -1 if $\lambda<0$. For later discussion we note that if $\alpha=\pi / 2$, i.e, $K(\pi / 2)$ is a half space then (1.5) gives

$$
\lambda_{1}=1, \quad \lambda_{2}=(1 / 3)\left(p-3-2 \sqrt{p^{2}-3 p+3}\right) /(p-1)
$$

$\left(\lambda_{1}(\pi / 2)=1\right.$ for $n \geq 2$ since $x_{1}=r \cos \theta$ is $p$ harmonic for $\left.1<p<\infty\right)$. Also if $\alpha=\pi, n=2$, i.e, $K(\pi)=\mathbb{R}^{2} \backslash(-\infty, 0]$, then (1.5) yields

$$
\lambda_{1}=1-1 / p, \lambda_{2}=(1 / 16)\left(7 p-16-\sqrt{81 p^{2}-288 p+288}\right) /(p-1) .
$$

For other values of $\lambda_{2}(\alpha)$ when $n=2$, see [22]. For $n \geq 3, \alpha=\pi / 2$, and $p=2$, one can use the Kelvin transformation to get $\lambda_{2}(\pi / 2)=1-n$ while if $p=n$, it follows from conformal invariance of the $n-$ Laplacian that $\lambda_{2}(\pi / 2)=-1$. Also if $p=(4 n-2) / 3$ then

$$
-2 \lambda_{2}(\pi)=\frac{p+1-n}{p-1}=\beta=\frac{n+1}{4 n-5} \text { since } u(r, \theta)=r^{-\beta / 2}(\cos (\theta / 2))^{\beta}
$$

in (1.3) for $\alpha=\pi$, as we first found using Maple and Mathematica, and then by direct calculation (see the discussion following Theorem 1.1). DeBlassie and Smits in [6] obtained estimates on $-\lambda_{2}(\pi / 2), 1<p<\infty, p \neq 2$, by leaving out the cotangent term in (1.4). In fact their solution to the DE in (1.4) with the cotangent term omitted leads to a super solution of the form (1.3) for the $p$ - Laplace equation, so leads to a upper estimate for $-\lambda_{2}(\pi / 2)$ in the $p$ - harmonic equation. Upper and lower estimates for $\lambda_{2}(\pi / 2)$, were also obtained by these authors in [7]. Recently, Llorente, Manfredi, Troy, and $\mathrm{Wu}$ in [21] used shooting methods to get upper and lower bounds for $\lambda_{2}(\pi / 2)$. These authors also gave a strictly ODE proof for existence of a solution in this case.

In [3] we prove

Theorem 1.1. Existence and uniqueness of $u_{i}=r^{\lambda_{i}} \phi_{i}, i=1,2$ also hold when $\alpha=\pi, n-1<p<\infty$. Moreover, $\lambda_{1}(\pi)=1-(n-1) / p$ for $n \geq 3$ and $n-1<p<\infty$. In fact as $\alpha \rightarrow \pi, \lambda_{1}(\alpha)-1+(n-1) / p \approx(\pi-\alpha)^{\frac{p+1-n}{p-1}}$.

Here $\approx$ means the ratio of the two functions is bounded above and below by constants depending only on $p$ and $n$. We note that if $1<p \leq n-1$, then a slit has $p-$ capacity zero in $\mathbb{R}^{n}, n \geq 3$, and so there are no solutions to (1.4). In fact Krol' and Maz'ya in the paper mentioned earlier obtained that as $\alpha \rightarrow \pi$,

$$
\lambda_{1}(\alpha) \approx\left\{\begin{array}{l}
(\pi-\alpha)^{\frac{n-1-p}{p-1}} \text { for } 1<p<n-1 \\
-\frac{1}{\log (\pi-\alpha)} \text { for } p=n-1
\end{array}\right.
$$

We also point out that Theorem 1 and conformal invariance of the $n$ - Laplacian imply for $p=n$ that $\lambda_{2}(\pi)=-1 / n$.

To outline our efforts in proving Theorem 1, we began by trying to use the DE in (1.4) to solve for $\lambda_{1}(\pi), \psi$. From a boundary Harnack inequality in [19] (see Theorem 1.9 and Lemma 5.3), we knew that 


$$
\lim _{\theta \rightarrow \pi} \psi(\theta)(\pi-\theta)=-\beta
$$

where $\beta=\frac{1+p-n}{p-1}$. Since $\phi$ has a relative maximum at $\theta=0$, it also followed that $\psi(0)=0$. Using these initial conditions, we first assumed for certain $p, n$ that

$$
\phi(\theta)=\cos (\theta / 2)^{\beta} e^{g(\cos (\theta / 2))} \text { for } 0 \leq \theta \leq \pi .
$$

To test the validity of what was then a conjecture, we considered as a test case $n=3, p=5 / 2,\left(\right.$ so $\left.\lambda_{1}(\pi)=1 / 5 ? ?\right)$ and in (1.6) put

$$
g(\cos (\theta / 2))=\sum_{k=0}^{\infty} a_{k}(\cos (\theta / 2))^{2 k}, 0 \leq \theta \leq \pi,
$$

where $a_{k}, k=1,2, \ldots$ are constants. Using this expression for $g$, our initial conditions, and then computing $\psi$ in (1.4), we deduced that the coeficients $a_{k}$, could be computed recursively. Thanks to Maple and Mathematicia, we were able to compute $a_{1}-a_{10}$. Using the resulting partial sum for $g$, and then computing $\psi$ we received strong evidence that $\lambda_{1}(\pi)=1 / 5$ in this test case. Later we put $x=\cos (\theta / 2), h(x)=$ $\beta+x g^{\prime}(x), x \in(-1,1)$, where $g$ is as in (1.6), and transformed (1.4) into,

$$
\begin{aligned}
& \left(1-x^{2}\right) x h^{\prime}(x)\left[(p-1)\left(1-x^{2}\right) h^{2}(x)+4 x^{2} \lambda^{2}\right]= \\
& -(p-1)\left(1-x^{2}\right)^{2} h^{4}(x)+\left[(p-1)+(n-2)\left(2 x^{2}-1\right)\right]\left(1-x^{2}\right) h^{3}(x) \\
& -4 \lambda[2 \lambda(p-1)+(n-p)] x^{2}\left(1-x^{2}\right) h^{2}(x)+ \\
& 4 \lambda^{2}\left[1+(n-2)\left(2 x^{2}-1\right)\right] x^{2} h(x)-16 \lambda^{3}[\lambda(p-1)+(n-p)] x^{4}
\end{aligned}
$$

where $h(0)=\beta$ and $\sqrt{1-x^{2}} h(x) \rightarrow 0$ as $x \rightarrow 1$. Expanding $h$ in a Macclaurin series and using Maple and Mathematica, we received even stronger evidence of the validity of $\lambda_{1}(\pi)=1 / 5$ when $n=3, p=5 / 2$. We also used this approach to check other values of our conjecture. However this approach seemed hopeless for proving Theorem 1. Finally we hit on using the following finess type proof.

\section{Outline of the Proof of Theorem 1 for $\lambda_{1}(\pi)$ When $n-1<p<n$}

To outline the proof of Theorem 1 we need some notation. Let

$$
B(z, \rho)=\left\{y=\left(y_{1}, \ldots, y_{n}\right) \in \mathbb{R}^{n}:|z-y|<\rho\right\} \text { whenever } z \in \mathbb{R}^{n}, \rho>0,
$$

and let $e_{1}=(1,0, \ldots, 0)$. Let $\mathcal{H}^{n-1}$ denote $n-1$ dimensional Hausdorff measure on $\mathbb{R}^{n}$ and let $d\left(F_{1}, F_{2}\right)$ denote the distance between the sets $F_{1}, F_{2}$. We write $d(x, F)$ for $d(\{x\}, F)$. Set $\mathbb{S}^{n-1}=\left\{x \in \mathbb{R}^{n}:|x|=1\right\}$ and let $c$ denote a positive constant $\geq 1$, which unless otherwise stated may only depend on $p, n, \alpha$.

Existence of a positive $p$ - harmonic function $v$ in $K(\alpha), 0<\alpha \leq \pi$, with $v\left(e_{1}\right)=1$ and continuous boundary value 0 on $\partial K(\alpha)$, follows easily from interior regularity results, the Dirichlet problem, and Wiener type estimates for $p$ harmonic functions given in [9]. For example given $1<p<\infty, \pi / 2 \leq \alpha<\pi$, and $l=1,2, \ldots$, let $v_{l}$ be 
the continuous function in $\bar{B}(0,2 l)$ with $v_{l}$ a $p$ - harmonic function in $B(0,2 l) \backslash\left[\left(\mathbb{R}^{n} \backslash\right.\right.$ $K(\alpha)) \cap \bar{B}(0, l)]$ and $v_{l} \equiv 0$ on $\left(\mathbb{R}^{n} \backslash K(\alpha)\right) \cap \bar{B}(0, l)$ while $v_{l}=M_{l}$ on $\partial B(0,2 l)$. Also $M_{l}$ is chosen so that $v_{l}\left(e_{1}\right)=1$. Using results which can be found in [9], one can show that a certain subsequence of $\left\{v_{l}\right\}_{l \geq 1}$, converges uniformly to $v \geq 0$, a Hölder continuous function on $\mathbb{R}^{n}$ which is $p$-harmonic in $K(\alpha)$ with $v \equiv 0$ on $\partial K(\alpha)$ and $v\left(e_{1}\right)=1$. The same argument gives a solution in $K(\pi)$ provided $p>n-1$. To prove existence of $v$ with the above properties in $K(\alpha)$ when $0<\alpha<\pi / 2$, let $v_{l}$ be the continuous function in $\bar{B}(0,2 l)$ with $v_{l}$ a $p$ - harmonic function in $B(0,2 l) \cap\left(K(\alpha) \backslash \bar{B}\left(l e_{1}, l \alpha / 8\right)\right), v_{l}\left(e_{1}\right)=$ 1 , and $v_{l} \equiv M_{l}$ on $\bar{B}\left(l e_{1}, l \alpha / 8\right)$ while $v_{l} \equiv 0$ on $[B(0,2 l) \backslash K(\alpha)] \cup \partial B(0,2 l)$. Taking limits as above, we get $v$.

Uniqueness of $v$ with the above properties, can be shown using boundary Harnack inequalities proved by Lewis and Nyström in [16], [19]. Indeed in [16], Theorem 2, the authors proved a boundary Harnack theorem for domains with a Lipschitz boundary which tailored to $K(\alpha), 0<\alpha<\pi$, is stated as follows:

Lemma 2.2. Let $\alpha \in(0, \pi), \rho>0, p$ fixed, $1<p<\infty$, and let $0<v_{1}, v_{2}$, be $p-$ harmonic in $K(\alpha) \cap B(0, \rho)$ with continuous boundary values and $v_{1}=v_{2} \equiv 0$ on $\partial K(\alpha) \cap B(0, \rho)$. There exists $c_{+} \geq 1, \sigma \in(0,1)$, depending only on $\alpha, n, p$, such that if $\rho^{+}=\rho / c^{+}$, and $x, y \in B\left(0, \rho^{+}\right) \cap K(\alpha)$, then

$$
\left|\frac{v_{1}(x)}{v_{2}(x)}-\frac{v_{1}(y)}{v_{2}(y)}\right| \leq c_{+}\left(\frac{|x-y|}{\rho^{+}}\right)^{\sigma} \frac{v_{1}(x)}{v_{2}(x)} .
$$

Letting $\rho \rightarrow \infty$ in Lemma 2.2, it follows that if $v=v_{1}$ and $v_{2}$ are positive $p-$ harmonic functions in $K(\alpha)$ with continuous boundary value 0 on $\partial K(\alpha)$, then $v / v_{2} \equiv$ constant. To prove that $v$ has the form (1.3) observe that for fixed $t>0$, the function $x \rightarrow v(t x), x \in K(\alpha)$, is positive, $p$ - harmonic, and has boundary value 0 on $\partial K(\alpha)$, so by uniqueness of $v$, we have

$$
v(t x)=v\left(t e_{1}\right) v(x), x \in K(\alpha) .
$$

Differentiating (2.8) with respect to $t$ and evaluating at $t=1$ we see that

$$
\langle x, \nabla v(x)\rangle=\left\langle e_{1}, \nabla v\left(e_{1}\right)\right\rangle v(x) \quad \text { whenever } \quad x \in K(\alpha) .
$$

If we put $\rho=|x|, x /|x|=\omega \in \mathbb{S}^{n-1}$, in this identity we obtain that

$$
\rho v_{\rho}(\rho \omega)=\left\langle e_{1}, \nabla v\left(e_{1}\right)\right\rangle v(\rho \omega) .
$$

Dividing this equality by $\rho v(\rho \omega)$ and integrating with respect to $\rho$ over $(0, r)$ we find that $v(r \omega)=r^{\lambda} v(\omega)$ whenever $\omega \in \mathbb{S}^{n-1}$ where $\lambda=\left\langle e_{1}, \nabla v\left(e_{1}\right)\right\rangle$. Finally since $p$ - harmonic functions are invariant under rotation it follows from this equality and uniqueness that $v$ has the form (1.3).

To prove uniqueness of $v$ in $K(\pi)$, we use arguments from [19], section 4, to prove (2.7) when $p>n-1$ and $v_{1}, v_{2}$ are positive $p$ - harmonic functions in $K(\pi)$ with continuous boundary value 0 on $\partial K(\pi)$. Uniqueness of $v$ implies, as in the argument following (2.8), that $v$ has the form (1.3). Existence, uniqueness, and showing $u$ has the form (1.3) when $\lambda<0$ in the so called Martin problem for $K(\alpha)$ is proved similarly (see [16] Corollary 5.25, for the analogue of Lemma 2.2). We omit the details. 
To avoid confusion in the rest of the proof of Theorem 1 we shall often write $u(\cdot, \alpha)$ for the positive $p$ - harmonic function in (1.3) with $u(\cdot, \alpha) \equiv 0$ on $\mathbb{R}^{n} \backslash K(\alpha), u\left(e_{1}, \alpha\right)=$ 1 , when $\alpha \in(0, \pi]$ and $p$ is fixed, $n-1<p<\infty$. We also write $\lambda(\alpha)$ for the eigenvalue, $\lambda_{1}$, in (1.3). From the maximum principle for $p-$ harmonic functions it follows that if $0<\alpha_{1}<\alpha_{2} \leq \pi$, then $u\left(\cdot, \alpha_{1}\right) \leq c u\left(\cdot, \alpha_{2}\right)$ in $K\left(\alpha_{1}\right) \cap B(0,1)$ so necessarily, $\lambda\left(\alpha_{2}\right) \leq \lambda\left(\alpha_{1}\right)$. Also strict inequality must hold since otherwise from (1.3) it would follow that $u\left(\cdot, \alpha_{1}\right) / u\left(\cdot, \alpha_{2}\right)$ has an absolute maximum in $K\left(\alpha_{1}\right)$, which again leads to a contradiction by way of the maximum principle for $p$ harmonic functions. From regularity estimates in [9] it follows that $u(\cdot, \alpha)$ converges uniformly to $u(\cdot, \pi)$ on $\mathbb{R}^{n}$ when $\alpha \rightarrow \pi$. Thus

$$
\lim _{\alpha \rightarrow \pi} \lambda(\alpha)=\lambda(\pi) \text { and } \lambda(\alpha)>\lambda(\pi) \text { when } \alpha \in(0, \pi) .
$$

To complete our proof of Theorem 1 we shall need some more notation, definitions, and lemmas. We begin with the following definition.

Definition 2.3. A bounded domain $D \subset \mathbb{R}^{n}$ is said to be starlike Lipschitz with respect to $z \in D$ provided

$$
\begin{aligned}
\partial D=\{z+\mathcal{R}(\omega) \omega & : \omega \in \partial B(0,1)\} \\
& \text { where } \log \mathcal{R}: \partial B(0,1) \rightarrow \mathbb{R} \text { is Lipschitz on } \partial B(0,1) .
\end{aligned}
$$

Under the above scenario we say that $z$ is the center of D. Let $\|\log \mathcal{R}\|_{\mathbb{S}^{n-1}}$ denote the Lipschitz norm of $\log \mathcal{R}$. We refer to $\|\log \mathcal{R}\|_{\mathbb{S}^{n-1}}$ as the starlike Lipschitz constant for $D$.

In [18], Theorem 3, Lewis and Nyström prove

Lemma 2.4. Let $D$ be a starlike Lipschitz domain with center at $z, w \in \partial D, 0<r<$ $|w-z| / 10$, and $p$ fixed, $1<p<\infty$. Let $v$ be $p-$ harmonic in $D \cap B(w, 4 r)$ with continuous boundary value $v \equiv 0$ on $\partial D \cap B(w, r)$. There exists $c_{\star} \geq 1$, depending only on $n, p$, and the starlike Lipschitz constant for $D$, such that if $\tilde{r}=r / c_{\star}$, then

(a) $\quad c_{\star}^{-1} \frac{v(x)}{d(x, \partial D)} \leq|\nabla v(x)| \leq c_{\star} \frac{v(x)}{d(x, \partial D)}, x \in D \cap B(w, \tilde{r})$,

(b) $\lim _{x \rightarrow y} \nabla v(x)=\nabla v(y)$ exists nontangentially for $\mathcal{H}^{n-1}$-almost every $y \in \partial D \cap B(w, \tilde{r})$,

(c) $\quad \nabla v(y)=-|\nabla v(y)| \nu(y)$ for $\mathcal{H}^{n-1}$ almost every $y \in \partial D \cap B(w, \tilde{r})$ where $\nu(y)$ is the unit outer normal to $\partial D(\alpha)$ at $y$.

(d) There exists $q>p$ and $c_{\star \star}$ with the same dependence as $c_{\star}$ such that

$$
\begin{aligned}
& \int_{\partial D \cap B(w, \tilde{r})}|\nabla v|^{q} d \mathcal{H}^{n-1} \leq c_{\star \star} r^{n-1-q} v\left(w^{\prime}\right)^{q} \text { where }\left|w-w^{\prime}\right|=\tilde{r} / 4 \text { and } \\
& w^{\prime} \text { lies on the ray from } z \text { to } w .
\end{aligned}
$$


Next if $1<p<n$, let

$$
F(x)=c_{p}|x|^{(p-n) /(p-1)} .
$$

Here $c_{p}=\frac{p-1}{n-p} \omega_{n}^{1 /(1-p)}$ where $\mathcal{H}^{n-1}\left(\mathbb{S}^{n-1}\right)=\omega_{n} \quad$ Then as is easily checked,

$$
\int_{\mathbb{R}^{n}}\left\langle|\nabla F|^{p-2} \nabla F, \nabla k\right\rangle d x=k(0), \text { whenever } k \in C_{0}^{\infty}\left(\mathbb{R}^{n}\right) .
$$

$F$ is said to be a Fundamental solution to the $p$ - Laplace equation with pole at 0 .

Definition 2.5. $\quad$ Given a starlike Lipschitz domain $D$ with center $z$ we say that $G$ is Green's function for the $p$ - Laplace equation in D, with pole at z provided

(a) $G$ is $p$-harmonic in $D \backslash\{z\}$,

(b) G has continuous boundary value 0 on $\partial D$,

(c) $F(x-z)=G(x)+\zeta(x), x \in D \backslash\{z\}$, where $0 \leq \zeta$ is bounded and Hölder continuous in $D$,

(d) $\quad \int_{D}\left\langle|\nabla G|^{p-2} \nabla G, \nabla \theta\right\rangle d \mathcal{H}^{n-1}=\theta(z)$ whenever $\theta \in C_{0}^{\infty}(D)$.

For existence and uniqueness of $G$ satisfying (2.10) see Lemma 10.4 in [1]. In [1] we use Lemma 2.4 to prove the following Rellich type equality for $1<p<n$,

Lemma 2.6. Let $D$ be a starlike Lipschitz domain with center at $z$, and for fixed $p, 1<p<n$, let $G$ be the Green's function for the $p$ - Laplace equation in $D$ with pole at $z$. Then

$$
\int_{\partial D}|\nabla G(x)|^{p}\langle x-z, \nu\rangle d \mathcal{H}^{n-1}=\frac{(n-p)}{p-1} \zeta(z)>0
$$

where $\nu$ is the outer unit normal to $\partial D$.

For $p=2$ this inequality was proved by Jerison and Kenig in [JK].

We now return to our proof of Theorem 1.1. For a fixed $p, n-1<p<n$, let $G_{1}$ denote the Green's function for $D_{1}=B(0,2) \cap K(\alpha), z=(1,0 \ldots, 0)=e_{1}$, and $0<\pi-\alpha<\pi / 4$. Also let $G_{2}$ denote the Green's function for $B(0,2)$ with pole at $e_{1}$. With this notation we state :

Lemma 2.7. For some $\tilde{c} \geq 1$, depending only on $p, n$,

$$
\int_{\partial K(\alpha) \cap B(0,2)}\left|\nabla G_{1}(x)\right|^{p}\left\langle x-e_{1}, \nu\right\rangle d \mathcal{H}^{n-1} \geq \frac{n-p}{p-1}\left(\zeta_{1}-\zeta_{2}\right)\left(e_{1}\right) \geq \tilde{c}^{-1}
$$

where $\nu$ is the outer unit normal to $\partial K(\alpha)$ and $\zeta_{1}, \zeta_{2}$, are defined relative to $G_{1}, G_{2}$, as in (2.10) (c). 
To prove the key inequality in (2.12) we first use (2.11) for $G_{1}, D_{1}$. Next we note that $\left|\nabla G_{1}\right| \leq\left|\nabla G_{2}\right|$ on $\partial B(0,2) \cap \partial D_{1}$ as follows from the Hopf boundary maximum principle. Using this note and (2.11) for $G_{2}, B(0,2)$, we get the left-hand inequality in (2.12). To prove the right-hand inequality in (2.12) we note that $G_{2} \approx 1$ in $B(0,1 / 4)$. Also if we put $G_{1} \equiv 0$ in $B(0,1 / 4) \backslash K(\alpha)$, then from Wiener type estimates for the $p$ - Laplace equation (using the fact that a slit has positive $p$ - capacity when $n-1<p<n$, see [23]), it follows that $G_{1}$ is Hölder continuous in $B(0,1 / 4)$ with Hölder exponent and norm independent of $\alpha \in(3 \pi / 4, \pi)$. Thus $G_{2}-G_{1}=\zeta_{1}-\zeta_{2} \geq$ a constant independent of $\alpha$ in $B(0,2 \rho)$ for some $0<\rho<1 / 8$. Also one can show that $G_{2}-G_{1}$ satisfies locally a uniformly elliptic PDE in divergence form. Using Harnack's inequality for positive solutions to this PDE we can connect a point in $K(\alpha) \cap B(0, \rho)$ to $e_{1}$ by a chain of balls with radii $\geq c^{-1}=c(p, n)^{-1}$, and then apply Harnack's inequality in successive balls to finally get the right-hand side of (2.12). The idea to use a Rellich type inequality to make estimates as above we garnered from a paper of Venouziou and Verchota in [28].

In order to use Lemma 2.7 in the proof of Theorem 1.1, first observe that $\bar{c} u(\cdot, \alpha) \geq$ $G_{1}$ on $D_{1} \backslash B\left(e_{1}, 1 / 2\right)$ where $\bar{c}=\bar{c}(p, n) \geq 1$ is independent of $\alpha \in(3 \pi / 4, \pi)$ so by the Hopf maximum principle

$$
\bar{c}|\nabla u(\cdot, \alpha)| \geq|\nabla G| \text { on } \partial K(\alpha) \cap B(0,2)
$$

and from the boundary Harnack inequality in Theorem 1.9 of [19], mentioned in the display above (1.6) we have

$$
|\nabla u(\cdot, \alpha)| \leq \hat{c}(\pi-\alpha)^{(2-n) /(p-1)} \text { on } \partial K(\alpha) \cap[B(0,2) \backslash B(0,1)]
$$

where $\hat{c} \geq 1$ depends only on $p, n$ when $\alpha \in[3 \pi / 4, \pi]$. Finally note that $\left\langle x-e_{1}, \nu\right\rangle=$ $\sin (\pi-\alpha)$ on $\partial K(\alpha) \cap B(0,2)$. Using this note and (2.13), (2.14), in (2.12) we conclude in view of (1.3) that for some $\breve{c}$ depending only on $p, n$ that

$$
\begin{aligned}
& \tilde{c}^{-1} \leq \int_{\partial K(\alpha) \cap B(0,2)} \sin (\pi-\alpha)|\nabla G|^{p} d \mathcal{H}^{n-1} \\
& \leq \breve{c}\left(\int_{0}^{2} r^{(\lambda(\alpha)-1) p+n-2} d r\right)(\pi-\alpha)^{\frac{p-n+1}{p-1}} \\
& \leq \frac{\breve{c}^{2}}{(\lambda(\alpha)-1) p+n-1}(\pi-\alpha)^{\frac{p-n+1}{p-1}} .
\end{aligned}
$$

where we have also used the fact that an element of surface area on $\partial S(\alpha)$ is of the form $\sin (\pi-\alpha)^{n-2} r^{n-2} d r$. From (2.15) and some arithmetic we conclude in view of (2.9) that

$$
\lambda(\pi)<\lambda(\alpha) \leq 1-(n-1) / p+c^{*}(\pi-\alpha)^{\frac{p-n+1}{p-1}} \text { as } \alpha \rightarrow \pi .
$$

for some $c^{*}=c^{*}(p, n)$ which gives the upper estimate for $\lambda_{1}(\alpha)$ in Theorem 1.1 when $n-1<p<n$. . The proof of the lower estimate for $\lambda_{1}(\alpha)$ as $\alpha \rightarrow \pi$ when $n-1<p<n$ is similar,just using Lemmas 2.6, 2.7, and Theorem 1.9 in [19], but somewhat more tedious to prove. This completes our outline of the proof of Theorem 
1.1 when $n-1<p<n$.

\section{Generalizations of Theorem 1}

If $O \subset \mathbb{R}^{n}$ is open and $1 \leq q<\infty$, let $W^{1, q}(O)$ denote the space of equivalence classes of functions $h$ with distributional gradient $\nabla h$, both of which are $q$-th power integrable on $O$.

Definition 3.8. For fixed $p>1, \delta \in(0,1)$, and $\mathcal{A}=\left(\mathcal{A}_{1}, \ldots, \mathcal{A}_{n}\right): \mathbb{R}^{n} \backslash\{0\} \rightarrow \mathbb{R}^{n}$ we say that $\mathcal{A} \in \mathcal{M}_{p}(\delta)$ provided $\mathcal{A}=\mathcal{A}(\eta)$ has continuous partial derivatives in $\eta_{k}, 1 \leq k \leq n$, and whenever $\xi \in \mathbb{R}^{n}, \eta \in \mathbb{R}^{n} \backslash\{0\}$,

$$
\begin{aligned}
& \text { (i) } \delta|\eta|^{p-2}|\xi|^{2} \leq \sum_{i, j=1}^{n} \frac{\partial \mathcal{A}_{i}}{\partial \eta_{j}} \xi_{i} \xi_{j} \text { and } \sum_{i=1}^{n}\left|\nabla \mathcal{A}_{i}(\eta)\right| \leq \delta^{-1}|\eta|^{p-2} \\
& \text { (ii) } \mathcal{A}(\eta)=|\eta|^{p-1} \mathcal{A}(\eta /|\eta|) \text {. }
\end{aligned}
$$

Definition 3.9. Given $\mathcal{A} \in \mathcal{M}_{p}(\delta)$, we say that $u$ is $\mathcal{A}$-harmonic in an open set $O$ provided $u \in W^{1, p}(\Omega)$ for each bounded open $\Omega$ with $\bar{\Omega} \subset O$ and

$$
\int\langle\mathcal{A}(\nabla u(y)), \nabla \theta(y)\rangle d y=0 \quad \text { whenever } \theta \in C_{0}^{\infty}(\Omega) .
$$

As a short notation for (3.17) we write $\nabla \cdot(\mathcal{A}(\nabla u))=0$ in $O$.

An important special class of $\mathcal{A}$ 's for us is when

(a) There exists $1 \leq \Lambda<\infty$ such that $\left|\frac{\partial \mathcal{A}_{i}}{\partial \eta_{j}}(\eta)-\frac{\partial \mathcal{A}_{i}}{\partial \eta_{j}^{\prime}}\left(\eta^{\prime}\right)\right| \leq \Lambda\left|\eta-\eta^{\prime}\right||\eta|^{p-3}$ whenever $0<\frac{1}{2}|\eta| \leq\left|\eta^{\prime}\right| \leq 2|\eta|$ and $1 \leq i, j \leq n$,

(b) $\mathcal{A}(\eta)=\mathbb{D} f(\eta)=\left(\frac{\partial f}{\partial \eta_{1}}, \frac{\partial f}{\partial \eta_{2}}, \ldots, \frac{\partial f}{\partial \eta_{n}}\right)$ so $f(t \eta)=t^{p} f(\eta)$ when $t>0$.

Note that if $\mathcal{A}=\mathbb{D} f$ in (3.18) and $f(\eta)=p^{-1}|\eta|^{p}$, then $u$ as in (3.17) is a weak solution to the $p$ - Laplace equation in $O$. Also observe that $\mathcal{A}$ - harmonic functions remain $\mathcal{A}$ harmonic under translation and dilation but not necessarily under rotations. We use the same notation as in Theorem 1.

Theorem 3.10. Given $\alpha \in(0, \pi), p \in(1, \infty)$, or $\alpha=\pi, p>n-1$, and $\mathcal{A} \in \mathcal{M}_{p}(\delta)$, there exist unique, $u_{i}$, for $i=1,2$, which are positive $\mathcal{A}$ - harmonic in $K(\alpha)$ with continuous boundary value 0 on $\partial K(\alpha) \backslash\{0\}, u_{i}\left(e_{1}\right)=1$, and of the form (1.2) with $\lambda_{2}(\alpha)<0<\lambda_{1}(\alpha)$.

Moreover if $\mathcal{A}$ also satisfies (3.18), then $\lambda_{1}(\pi)=1-(n-1) / p$ for $n-1<p<\infty$. In fact

$$
\lambda_{1}(\alpha)-1+(n-1) / p \approx(\pi-\alpha)^{\frac{p-n+1}{p-1}} \text { as } \alpha \rightarrow \pi .
$$

where ratio constants depend only on $p, n, \delta, \Lambda$. 


\subsection{Outline of the Proof of Theorem 3.10.}

Proof. Existence and uniqueness of $u_{i}, i=1,2$, in $K(\alpha), 0<\alpha \leq \pi$, follows from boundary Harnack inequalities proved in [15] for Reifenberg flat domains and arguments similar to those in section 4 of [19]. The proof that $\lambda_{1}(\pi)=1-(n-1) / p$ is essentially the same as the proof we outlined in the $p$ harmonic setting for $n-1<p<n$. Indeed Lemmas 2.2 2. 2.4 2.6, 2.7 are proved in Proposition 9.7, Lemma 10.9, Lemma 13.7, and display (13.86), respectively, of [1] in the $\mathcal{A}$ - harmonic setting when $\mathcal{A} \in \mathcal{M}_{p}(\delta), 1<p<n$, satisfies (3.18).

Remark 3.11. Lemma 2.6 remains valid when $p \geq n$, for $G$ properly defined. However for $p>n$ there is a sign reversal in the inequality and so this Lemma can no longer be used to get an analogue of Lemma 2.7 when $p \geq n$. Instead in [3] we use a different Rellich inequality derived from the work on Theorem B in [1] and Theorem $B$ in [2] on a Minkowski existence problem. Armed with this inequality, the proof of Theorems 1.1, 3.10 are similar to the proof outlined for $n-1<p<n$. Finally we note that our interest in this eigenvalue problem stems from our study of regularity in a Minkowski problem, originally proved in Theorem 0.7 of [10] for harmonic functions and later generalized in Theorem 1.4 of [5] to $p$-harmonic functions when $1<p<2$.

\section{REFERENCES}

[1] Akman, M., Gong, J., Hineman, J., Lewis, J., And Vogel, A. The Brunn-Minkowski inequality and A Minkowski problem for nonlinear capacity. To appear in Memoirs of the AMS, arXiv:1709.00447 ((2017)). (Cited on pages 7 and 10.)

[2] Akman, M., Lewis, J., SaAri, O., And Vogel, A. The Brunn-Minkowski inequality and A Minkowski problem for $\mathcal{A}$-harmonic Green's function. Submitted, arXiv:1810.03752 (2018). (Cited on page 10.)

[3] Akman, M., Lewis, J., And Vogel, A. Note on an eigenvalue problem with applications to a Minkowski type regularity problem in $\mathbb{R}^{3}$. Work in progress (2018). (Cited on pages 3 and 10.)

[4] Aronsson, G. Construction of singular solutions to the $p$-harmonic equation and its limit equation for $p=\infty$. Manuscripta Math. 56, 2 (1986), 135-158. (Cited on page 2.)

[5] Colesanti, A., Nyström, K., Salani, P., Xiao, J., Yang, D., and Zhang, G. The Hadamard variational formula and the Minkowski problem for p-capacity. Adv. Math. 285 (2015), 1511-1588. (Cited on page 10.)

[6] DeBlassie, D., And Smits, R. G. The $p$-harmonic measure of a small spherical cap. Matematiche (Catania) 71, 1 (2016), 149-171. (Cited on page 3.)

[7] DeBlassie, D., And Smits, R. G. The p-Harmonic Measure of Small Axially Symmetric Sets. Potential Anal. 49, 4 (2018), 583-608. (Cited on page 3.)

[8] Gkikas, K. T., And VÉron, L. The spherical $p$-harmonic eigenvalue problem in non-smooth domains. J. Funct. Anal. 274, 4 (2018), 1155-1176. (Cited on page 2.)

[9] Heinonen, J., Kilpeläinen, T., And Martio, O. Nonlinear Potential Theory of Degenerate Elliptic Equations. Dover Publications Inc., 2006. (Cited on pages 4, 5, and 6.)

[10] Jerison, D. A Minkowski problem for electrostatic capacity. Acta Math. 176, 1 (1996), 1-47. (Cited on page 10.)

[11] Jerison, D. S., And Kenig, C. E. Boundary value problems on Lipschitz domains. In Studies in partial differential equations, vol. 23 of MAA Stud. Math. Math. Assoc. America, Washington, DC, 1982, pp. 1-68. (Not cited.) 
[12] Krol', I. N. The behavior of the solutions of a certain quasilinear equation near zero cusps of the boundary. Trudy Mat. Inst. Steklov. 125 (1973), 140-146, 233. Boundary value problems of mathematical physics, 8. (Cited on page 2.)

[13] Krol', I. N., AND MaZ'Ja, V. G. The lack of continuity and Hölder continuity of the solutions of a certain quasilinear equation. Zap. Naučn. Sem. Leningrad. Otdel. Mat. Inst. Steklov. (LOMI) 14 (1969), 89-91. (Cited on page 2.)

[14] Krol', I. N., And MaZ'Ja, V. G. The absence of the continuity and Hölder continuity of the solutions of quasilinear elliptic equations near a nonregular boundary. Trudy Moskov. Mat. Obšč. 26 (1972), 75-94. (Not cited.)

[15] Lewis, J., Lundström, N., And Nyström, K. Boundary Harnack inequalities for operators of $p$-Laplace type in Reifenberg flat domains. In Perspectives in partial differential equations, harmonic analysis and applications, vol. 79 of Proc. Sympos. Pure Math. Amer. Math. Soc., Providence, RI, 2008, pp. 229-266. (Cited on page 10.)

[16] Lewis, J., AND Nyström, K. Boundary behavior and the Martin boundary problem for $p$ harmonic functions in Lipschitz domains. Ann. of Math. (2) 172, 3 (2010), 1907-1948. (Cited on page 5.)

[17] Lewis, J. L. Smoothness of certain degenerate elliptic equations. Proc. Amer. Math. Soc. 80, 2 (1980), 259-265. (Cited on page 2.)

[18] Lewis, J. L., And Nyström, K. Boundary behaviour for $p$ harmonic functions in Lipschitz and starlike Lipschitz ring domains. Ann. Sci. École Norm. Sup. (4) 40, 5 (2007), 765-813. (Cited on page 6.)

[19] Lewis, J. L., And Nyström, K. Quasi-linear PDEs and low-dimensional sets. J. Eur. Math. Soc. (JEMS) 20, 7 (2018), 1689-1746. (Cited on pages 3, 5, 8, and 10.)

[20] Lewis, J. L., And Vogel, A. On p Laplace polynomial solutions. J. Anal. 24, 1 (2016), 143-166. (Cited on page 2.)

[21] Llorente, J. G., Manfredi, J. J., Troy, W. C., And Wu, J.-M. On p-harmonic measures in half spaces. Preprint, arXiv:1807.10367 (2018). (Cited on page 3.)

[22] Lundström, N. L. P., And Vasilis, J. Decay of a $p$-harmonic measure in the plane. Ann. Acad. Sci. Fenn. Math. 38, 1 (2013), 351-366. (Cited on page 3.)

[23] MaZ'ya, V. Sobolev spaces with applications to elliptic partial differential equations, augmented ed., vol. 342 of Grundlehren der Mathematischen Wissenschaften [Fundamental Principles of Mathematical Sciences]. Springer, Heidelberg, 2011. (Cited on page 8.)

[24] Porretta, A., And Véron, L. Separable $p$-harmonic functions in a cone and related quasilinear equations on manifolds. J. Eur. Math. Soc. (JEMS) 11, 6 (2009), 1285-1305. (Cited on page 2.)

[25] Tkachev, V. G. On the non-vanishing property for real analytic solutions of the $p$-Laplace equation. Proc. Amer. Math. Soc. 144, 6 (2016), 2375-2382. (Cited on page 2.)

[26] Tkachev, V. G. New explicit solutions to the $p$-laplace equation based on isoparametric foliations. Preprint, arXiv:1802.09892 (2018). (Cited on page 2.)

[27] Tolksdorf, P. On the dirichletproblem for quasilinear equations. Communications in Partial Differential Equations 8, 7 (1983), 773-817. (Cited on page 2.)

[28] Venouziou, M., and Verchota, G. C. The mixed problem for harmonic functions in polyhedra of $\mathbb{R}^{3}$. In Perspectives in partial differential equations, harmonic analysis and applications, vol. 79 of Proc. Sympos. Pure Math. Amer. Math. Soc., Providence, RI, 2008, pp. 407-423. (Cited on page 8.) 
Murat Akman, Department of Mathematics, University of Connecticut, Storrs, CT 06269-1009

Email address: murat.akman@uconn.edu

URL: http://www. math.uconn.edu/ akman/

John Lewis, Department of Mathematics, University of Kentucky, Lexington, KenTUCKY, 40506

Email address: johnl@uky.edu

$U R L:$ http: //www.ms.uky.edu/ johnl/

Andrew Vogel, Department of Mathematics, Syracuse University, Syracuse, New YORK 13244

Email address: alvogel@syracuse.edu 